\title{
Droplet Digital PCR Based Detection of EGFR Mutations in Advanced Lung Cancer Patient Liquid Biopsies: A Comparison of Circulating Tumour DNA Extraction Kits
}

Pei N Ding ${ }^{1,2,3 \#}$, Yafeng F Ma1,4\#, Tara J Roberts ${ }^{1,2,4}$, Wei Chua ${ }^{1,3}$, Victoria J Bray ${ }^{3,4}$, David Lynch ${ }^{1,2}$, Daniel Burke ${ }^{5}$, Paul de Souza ${ }^{1,2,3,4}$ and Therese M Becker ${ }^{1,2,4^{*}}$

${ }^{1}$ Centre for Circulating Tumour Cell Diagnostics \& Research at Ingham Institute for Applied Medical Research, Campbell St, Liverpool NSW, Australia ${ }^{2}$ School of Medicine, Western Sydney University, Campbelltown NSW, Australia

${ }^{3}$ Liverpool Hospital, Elizabeth St \& Goulburn St, Liverpool NSW, Australia

${ }^{4}$ South Western Sydney Medical School, University of New South Wales, Goulburn St, Liverpool NSW, Australia

${ }^{5}$ National Measurement Institute (NMI), Lindfield, Sydney, New South Wales, Australia

"Equal contribution

\begin{abstract}
Background: Mutations in the epidermal growth factor receptor gene, EGFR, predict response or resistance to first generation tyrosine kinase inhibitors in non-small cell lung cancer. These biomarkers can now be conveniently detected from liquid biopsies, however technical details of these assays are still being refined.

Objective: To compare detection of four different non-small cell lung cancer (NSCLC) associated EGFR mutations from patient ctDNA isolated with five different ctDNA isolation kits.

Methods: Droplet digital PCR (ddPCR) assays detecting four EGFR mutations were developed. ctDNA was isolated with five kits from plasma samples, one pleural and one ascites fluid from nine NSCLC patients with known EGFR mutations. ctDNA fragment sizes and concentrations were also assessed.

Results: Each kit isolated DNA from all samples which contained an expected dominant DNA fragment of $\sim 170$ base pairs. Normalised for plasma input, one kit produced ctDNA extracts which consistently enabled the highest copy number detection for all EGFR variants, and importantly was able to validate mutations in all patient samples. Other kits stood out in regards to cost economy as well as ease and speed of processing but were less efficient and one kit was found to be incompatible with ddPCR.

Conclusion: This study demonstrated successful ctDNA isolation from plasma, pleural fluid and ascites by four of five ctDNA isolation kits. The QIAmp circulating nucleic acid kit produced consistently the most sensitive detection of EGFR variants. While other kits allow for lower volume plasma input down to $0.1 \mathrm{ml}$, are faster, more economical and simpler to use, they are challenged by very low ctDNA concentrations in plasma.
\end{abstract}

Keywords: ctDNA; cfDNA; Biomarker; Lung cancer; EGFR mutation; Droplet digital PCR

\section{Introduction}

Cell free DNA (cfDNA) can be released into the blood stream during apoptotic cell death and tissue turn over. Circulating tumour DNA (ctDNA) represents a variable proportion of cancer tissue derived cfDNA in cancer patients (range from $0.01 \%$ to over 90\%) [1,2].

In recent years, it has been recognised that isolation and analysis of ctDNA from simple blood samples (liquid biopsies) permits tumour biomarker detection. Compared to tissue biopsy, liquid biopsies can be screened for tumour biomarkers in a low cost and non-invasive manner. Further, easy access and repeated sampling, as well as rapid turnaround times for biomarker identification and validation makes liquid biopsy a feasible strategy to regularly sample tumour biomarker information $[1,3]$. Evidence shows that quantification of ctDNA by detecting tumour specific genetic variants may predict prognosis and tumour response to treatment in solid tumours [4-7]. In the field of lung cancer, osimertinib, a third generation EGFR inhibitor, was recently FDA and Therapeutics Good Administration (TGA) approved for treatment of patients with EGFR mutated NSCLC, including patients who have acquired the EGFR-T790M resistance mutation against first generation inhibitors [8]. Through large clinical trials, it has now been established that the detection of activating and drug-resistance EGFR mutations using ctDNA has high sensitivity and specificity compared with tissue biopsies. Patients treated with EGFR inhibitors based on ctDNA positivity for EGFR mutations have similar response rates compared with EGFR mutation positive tissue biopsy patients, providing a solid basis for recognition of the importance and utility of ctDNA in place of tissue biopsy for mutation testing to guide treatment decisions and monitoring response $[9,10]$.

However, in order for ctDNA testing to be implemented in the clinical setting, a reliable and efficient approach to allow high throughput methodology is necessary, and it is important to have a robust and efficient pre-analytical step of $\mathrm{cf} / \mathrm{ctDNA}$ isolation. Although comparisons of $\mathrm{cf} / \mathrm{ctDNA}$ extraction methods have been reported previously, often either no or limited tumour specific actionable

*Corresponding author: Therese M Becker, Centre for Circulating Tumour Cell Diagnostics \& Research at Ingham Institute for Applied Medical Research, 1 Campbell St Liverpool NSW 2170, Australia, Tel: +61-2-873 89033; Fax: +61-2 9602 9441; E-mail: t.becker@unsw.edu.au

Received July 07, 2018; Accepted August 04, 2018; Published August 07, 2018

Citation: Ding PN, Ma YF, Roberts TJ, Chua W, Bray VJ, et al. (2018) Drople Digital PCR Based Detection of EGFR Mutations in Advanced Lung Cancer Patient Liquid Biopsies: A Comparison of Circulating Tumour DNA Extraction Kits. J Mol Biomark Diagn 9: 397. doi: 10.4172/2155-9929.1000397

Copyright: $\odot 2018$ Ding PN, et al. This is an open-access article distributed under the terms of the Creative Commons Attribution License, which permits unrestricted use, distribution, and reproduction in any medium, provided the original author and source are credited. 
biomarkers were tested in patient or healthy control samples, making it difficult to clarify kit values for tumour biomarker detection [1117]. As part of a larger ongoing study of advanced EGFR mutant NSCLC patients, we have developed several sensitive droplet digital PCR (ddPCR) - based assays to accurately detect a range of different EGFR mutations. Here, we systematically compared five commercially available cfDNA extraction kits. We investigated whether the detectability of four different EGFR activating and resistance mutations was affected by using different cfDNA isolation kits, by evaluating copy number detection of mutant and wild type cf/ctDNA from plasma. We also evaluated DNA extraction from ascites and pleural fluid using the same kits.

\section{Material and Methods}

\section{Patient samples}

Nine patients with advanced, established EGFR-mutated NSCLC were prospectively enrolled with written informed consent and approval by the South Western Sydney Biosafety Committee (HREC/13/LPOOL/158). A total of eleven samples were available for analysis (nine plasma samples, and from two patient's additional ascites or pleural fluid was collected). All patients had been diagnosed with stage 4 lung cancer and had activating positive EGFR mutation based on tissue biopsy analysis by a National Association of Testing Authorities, Australia (NATA) accredited pathology laboratory. Two patients carried both EGFR activating and T790M resistance mutations. Patient and disease characteristics are summarized in Table 1.

\section{Plasma processing}

Two $9 \mathrm{ml}$ EDTA vacutubes (Greiner, Frickenhausen, Germany) were used to collect whole blood samples from each patient. All blood samples were processed within 2 hours from the time of blood collection. Plasma was separated from the cellular fraction initially by centrifugation at $800 \mathrm{~g}$ at room temperature for 10 minutes followed by second centrifugation of the supernatant at maximum microfuge speed $(13000 \mathrm{rpm})$ at $4^{\circ} \mathrm{C}$ for 10 minutes. Supernatant was stored aliquoted at $-80^{\circ} \mathrm{C}$ until ctDNA extraction. Pleural and ascites fluid were processed within 24 hours of collection time using the same method.

\section{Cell free DNA extraction}

Table 2 lists the five tested ctDNA isolation kits: QIAmp (QIA) circulating nuclei acid kit (Qiagen, Valencia, CA, USA), Plasma cellfree circulating DNA purification mini kit (NRG) (Norgen Biotek Corp., ON, Canada), Nucleospin (NS) plasma XS (Macherey-Nagel, Germany) and MagMAX (MM) cell-free total nucleic acid isolation kit (Thermofisher Scientific, Waltham, MA) and the NextPrep-Mag cfDNA isolation kit (BIOO Scientific) (Austin, TX, USA) (BIO). ctDNA was extracted according to the manufacturers' protocols.

\section{ctDNA concentration}

ctDNA concentration was measured using the Qubit doublestranded (ds) DNA HS Assay kit on a Qubit 4.0 Fluorometer (Invitrogen, Life Technologies, CA, USA) according to manufacturer's instructions.

\section{Bio-analyser DNA testing}

Bio-analyser data were obtained for selected plasma samples using the Agilent Bio-analyser 2100 High Sensitivity DNA kit (Agilent, Santa Clara, CA), based upon the manufacturer's protocol.

\section{Droplet digital PCR}

Primers and probes were generated to detect EGFR-L858R, -S768I,
-T790M and the corresponding wild type amplicons as well as the EGFR exon 19 deletion using the QX200 BioRad droplet digital PCR (ddPCR) suite (BioRad, Gladesville, Australia). The assays were optimised for annealing temperatures combined with BioRad's recommended primer and probe concentrations and standard droplet digital PCR (ddPCR) protocol (Supplementary Table 1). For all point mutation assays, biomarker (mutation) versus normal (wild type) detection were multiplexed as described before [18]. A double probe detection method that essentially follows the strategy by Oxnard et al., with adjusted primer and probe sequences was used to detect the exon 19 deletion EGFR biomarker (Supplementary Table 1 and Figure 1) [19]. Assay sensitivities were tested with DNA from EGFR mutant titrated into that from wild type cell lines as available (H1975: EGFR-L858R and EGFR-T790M; HCC827: EGFR exon 19 deletion, healthy donor peripheral blood lymphocytes: EGFR wild type) or for the EGFR-S768I variant with synthetic gene fragments (Integrated DNA Technologies, Singapore). Sensitivity of all assays was at least 1:1000 as determined by titration of mutant into wild type DNA. Assay specificities were tested using cfDNA from 14 healthy controls and metastatic NSCLC patients negative for EGFR mutations by tissue biopsy and was 100\% for all assays The QX200 droplet reader and Quanta Soft software V1.7.4 was used for analysis (Figure 1).

Detected mutant and wild type copy numbers were normalised based on the volumes of plasma/ascites/pleural fluid used for DNA extraction, elution and the input into the ddPCR reaction (DNA copies/ $\mathrm{ml}$ plasma) based on the following formula:

$\frac{\text { Events } \times 20}{\text { Input ctDNA volume }(\mu l)} \times \frac{\text { Elution volume }(\mu l)}{\text { Plasma volume }(\mu l)}$

\section{Statistical analysis}

The non-parametric comparison of cfDNA wild type or mutant copy numbers detected for the same plasma using different extraction kits, was evaluated using the Wilcoxon signed-rank test for paired data. Differences in DNA concentration for different kits were tested using Friedman test. Statistical analysis and graphs were generated in GraphPad Prism (version 6.01). A p-value of $p<0.05$ was considered statistically significant.

\section{Results}

\section{Isolation efficiency and DNA integrity}

ctDNA concentrations as measured by Qubit were of a relatively similar range regardless of extraction kit used with no significant variation of mean DNA concentration between the extracts from the five tested kits (Table 3). As shown by bio-analyser testing all kits produced a peak for ctDNA as expected around 170 base pairs. There was no obvious correlation between this peak's height and kit performance for ddPCR biomarker detection, while unexpectedly differences in peak heights of the included 35 and 10380 base pair reference markers was observed (Figure 2).

\section{Circulating DNA detection}

Despite evidence for DNA presence using Qubit DNA concentration measurements as well as the bio-analyser (Table 3 and Figure 2), in our hands the BIO kit repeatedly proved not suitable for ddPCR applications as extracted DNA produced extremely poor or no separation between positive ddPCR events and baseline "empty" events. This was consistent in our hands for all EGFR biomarkers tested in this study. Independently our team found the same result for reference genes and unrelated biomarkers from different patient plasma samples 
A

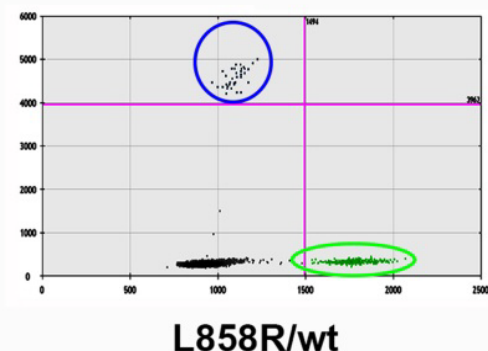

B

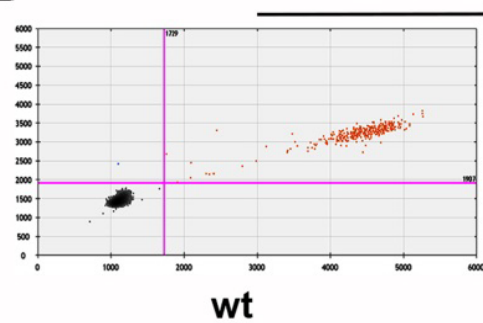

\section{EGFR point mutation assays}


EGFR exon 19 deletion assay
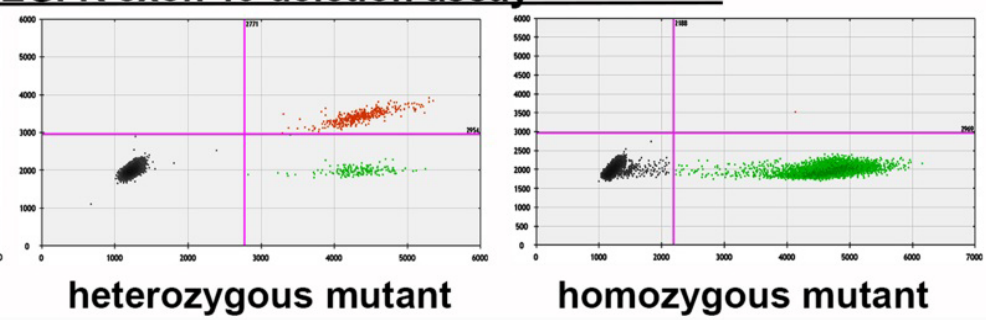

Figure 1: EGFR Biomarker Assays (A) EGFR point mutation ddPCR assays employ primers producing $65-86$ base pair amplicons comprising the mutation site. Wild type (wt) and mutant DNA amplicons are distinguished by specific TaqMan probes. Separation of wt DNA copies (green dots) from empty droplets/events (black dots) and mutant events (blue dots) is presented. (B) For the EGFR exon 19 deletion assay, primers amplify DNA incorporating the common deletion site and the assay has one probe complementary to consistently deleted sequences (only detected in wt DNA) and one probe to consistently retained sequences within the amplicon (detected in both mutant and wt DNA). Consequently, wt DNA copies are detected by the combination of both fluorescent markers (indicated by brown dots), while mutant DNA synthesis only involves one probe (indicated by green dots).

\begin{tabular}{|c|c|}
\hline $\begin{array}{c}\text { Clinical/disease characteristics } \\
\text { Median age (range) }\end{array}$ & $\begin{array}{c}\text { Number } \mathbf{n} \\
\mathbf{( \% )}\end{array}$ \\
\hline Gender $\mathbf{n = 9}$ & $59(41-74)$ \\
\hline Male & $3(33.3)$ \\
\hline Female & $6(66.7)$ \\
\hline EGFR Mutations, $\mathbf{n = 1 3 ^ { * }}$ & $3(13)$ \\
\hline L858R & $4(30.8)$ \\
\hline Exon 19 deletion & $2(15.4)$ \\
\hline S768I & $4(30.8)$ \\
\hline T790M & $9(82)$ \\
\hline Samples tested $\mathbf{n = 1 1}$ & $1(9)$ \\
\hline Plasma & $1(9)$ \\
\hline Pleural fluid & \\
\hline Ascitic fluid & \\
\hline Note: ${ }^{*}$ in 2 of 11 samples 2 EGFR mutations were validated independently
\end{tabular}

Table 1: Patient characteristics.

(data not shown). Since ddPCR assays are a major down-stream cfDNA analysis tool for our research, we excluded the BIO kit from further analysis. For detection of cfDNA by analysing the wild type EGFR amplicons, QIA performed, with an average detection of 5915 copies $/ \mathrm{ml}$ plasma, significantly better compared to the other three kits, averaging 2735 copies/ml (NRG, $p=0.004$ ), 2174 copies/ml (NS, $p=0.001$ ) and 3064 copies $/ \mathrm{ml}$ (MM, $p=0.002$ ). In fact, detected wild type copies after QIA extraction were consistently above the average detection for DNAs extracted with all four kits; while the other kits' performance was more variable. Nevertheless, wild type copies were detected in nearly all instances. Any DNA extractions without wild type amplicon detection were considered to have failed for uncertain reasons (Figure 3).

\section{EGFR mutation detection}

ddPCR was used to detect the presence of 3 different EGFR point mutations (L858R, S768I, T790M) and the exon 19 deletion. Existing EGFR mutations ( $\geq 1$ positive droplet) were validated in $10 / 13(76 \%)$ DNA extracts using the NRG kit, 11/13 (85\%) DNA samples using the NS kit, in 12/13 (92\%) DNA samples using the MM kit and in all 13 (100\%) samples using the QIA kit. In all but two samples, the QIA kit consistently extracted more ctDNA, as measured by ddPCR, compared with the other three kits (Figures 3 and 4). Importantly, when focusing on 5 patient plasma samples $(4,6,8,9$ and 10$)$ that detected low mutant copies when tested with QIA (ie. $<100$ copies/ml plasma), the NRG kit detected the biomarker in only $3(60 \%)$, the NS kit in $4(80 \%)$ and MM kit in $4(80 \%)$ of samples (Figure 4$)$.

\section{Discussion}

The aim of this study was to thoroughly compare five different ctDNA extraction kits for their efficiency in isolating quality DNA for biomarker testing using ddPCR applications.

ctDNA is present in very low concentrations in the blood, as little as $0.01 \%$ to up to $90 \%$ of approximately $22-3922 \mathrm{ng} / \mathrm{ml} \mathrm{cfDNA}$ and once blood is drawn ctDNA is subject to increasing dilution with normal DNA deriving from blood cell lysis in the blood sample over time [1,2]. Therefore it is important to have a highly efficient ctDNA extraction method to allow sensitive detection of biomarkers with low allelic frequency even in patients with low tumour burden or tumours that shed low amounts of nucleic acids into circulation. A method which is economical and could accommodate high throughput testing is highly desirable for ctDNA testing to be more readily incorporated in routine clinical settings. 
Citation: Ding PN, Ma YF, Roberts TJ, Chua W, Bray VJ, et al. (2018) Droplet Digital PCR Based Detection of EGFR Mutations in Advanced Lung Cancer Patient Liquid Biopsies: A Comparison of Circulating Tumour DNA Extraction Kits. J Mol Biomark Diagn 9: 397. doi: 10.4172/21559929.1000397

Page 4 of 6

\begin{tabular}{|c|c|c|c|c|c|c|c|}
\hline $\begin{array}{c}\text { Kit } \\
(\text { acronym*) }\end{array}$ & Manufacturer & Isolation matrix & Protocol & $\begin{array}{l}\text { Recommended Plasma } \\
\text { volume [ml] } \\
\text { (This study) }\end{array}$ & $\begin{array}{c}\text { Recommended Elution } \\
\text { volume }[\mu l] \\
\text { (This study) }\end{array}$ & $\begin{array}{l}\text { Process } \\
\text { Time [min] }\end{array}$ & $\begin{array}{c}\text { Cost/sample } \\
\text { [AUS\$] }\end{array}$ \\
\hline $\begin{array}{l}\text { QIAmp circulating nuclei } \\
\text { acid kit (QIA) }\end{array}$ & Qiagen & Silica column & $\begin{array}{c}\text { Manual/ } \\
\text { semi- automated }\end{array}$ & $\begin{array}{l}1-5 \\
(3)\end{array}$ & $\begin{array}{l}20-150 \\
(60)\end{array}$ & 120 & 30 \\
\hline $\begin{array}{l}\text { Plasma cell-free circulating } \\
\text { DNA purification mini kit } \\
\text { (NRG) }\end{array}$ & Norgen & Spin column & Manual & $\begin{array}{c}0.2-0.5 \\
(0.5)\end{array}$ & $\begin{array}{c}25-50 \\
(20)\end{array}$ & 20 & 7 \\
\hline $\begin{array}{c}\text { Nucleo spin plasma XS } \\
\text { (NS) }\end{array}$ & Macherey-Nagel & Silica column & Manual & $\begin{array}{l}0.72 \\
(0.5)\end{array}$ & $\begin{array}{l}5-30 \\
(15)\end{array}$ & 25 & 7 \\
\hline $\begin{array}{l}\text { Mag MAX cell-free total } \\
\text { nucleic acid isolation kit } \\
\text { (MM) }\end{array}$ & Thermo-fisher & Magnetic beads & Manual/automated & $\begin{array}{l}1-6 \\
(1)\end{array}$ & $\begin{array}{c}15-60 \\
(15)\end{array}$ & 90 & 20 \\
\hline $\begin{array}{l}\text { Next Prep-Mag } \\
\text { cfDNA isolation kit } \\
(\mathrm{BIO})\end{array}$ & Bioo Scientific & Magnetic beads & Manual & $\begin{array}{c}<1-3 \\
(0.2-0.6)\end{array}$ & $\begin{array}{l}0.012 x \text { input } \\
(10)\end{array}$ & 25 & 8 \\
\hline
\end{tabular}

Table 2: cfDNA extraction kits.

\begin{tabular}{|c|c|c|}
\hline Extraction kit & $\begin{array}{c}\text { Mean DNA } \\
\text { concentration }(\mathbf{n g} / \boldsymbol{\mu l})\end{array}$ & Range (ng/ $\mathbf{\mu l})$ \\
\hline NRG & 1.1 & $0.3-3.6$ \\
\hline NS & 0.3 & $0.2-0.5$ \\
\hline MM & 0.9 & $0.2-3.4$ \\
\hline QIA & 1.3 & $0.5-3.5$ \\
\hline BIO & 0.45 & $0-0.7$ \\
\hline
\end{tabular}

Table 3: Extracted cfDNA concentration ranges.



Figure 2: Dominant DNA species in isolated cfDNA is 170 base pairs. $1 \mu$ of DNA extracts from all kits were compared by bio-analyser. Representative DNA detection histograms for ctDNAs of patient sample 4 are depicted. The cfDNA characteristic peak of $\sim 170$ base pairs is indicated by arrows. MRK: DNA size marker.

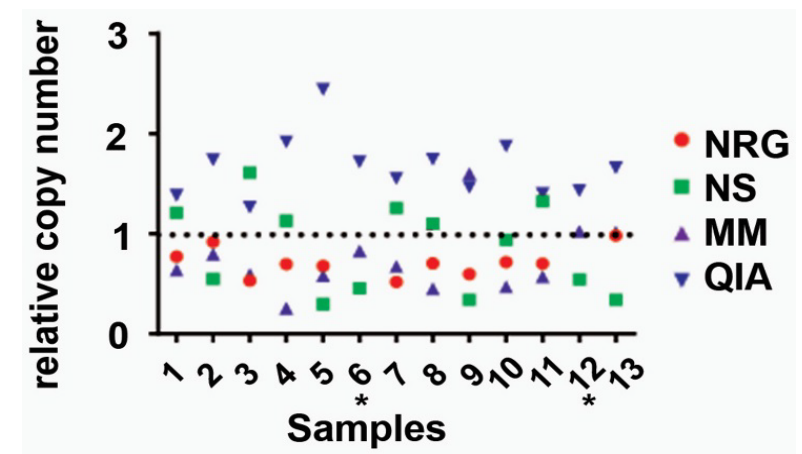

Figure 3: Wild type cfDNA detection by ddPCR. Mean wild type (wt) DNA copy numbers detected by ddPCR using the various EGFR assays differed for samples dependent on ctDNA extraction methods (range 2174 to 5915 copies). The graph illustrates wt copy number detection normalised per ml plasma (ascites or pleural fluid) input relative to mean copy numbers detected across all extractions (NRG, NS, MM, QIA) kits for each patient sample. * NRG DNA extracts considered failed due to lack of wt ctDNA detection by ddPCR.

Our study tested a cohort of samples from patients with advanced EGFR-mutated NSCLC, with in-house established EGFR mutation assays to detect all these biomarkers. We compared five different cfDNA extraction kits with different methods of isolation (column versus magnetic beads), comparing the most commonly used kit (QIA) with other commercially available kits. In addition to plasma samples our study also compared the performance of cfDNA isolation kits for DNA extraction from ascites and pleural fluid for biomarker detection, which has not been reported previously.

Notably, the tested kits require or allow for different ranges of plasma volume input, from as low as $200 \mu \mathrm{l} \mathrm{up} \mathrm{to} 10 \mathrm{ml}$. In cases where only limited volumes of plasma are available, the ability to accommodate lower input volumes may be an important consideration in selecting the most suitable ctDNA extraction kit. Some manufacturers also offer different kits that apparently use similar isolation chemistries for different input volumes, however, in our hands these kits do not necessarily deliver similar results (preliminary data not shown) which agrees with reports by others $[12,15,16]$.

Of the kits used in this study, the QIA kit uses the highest volume of plasma and is the most labour intensive with approximately 2 hours processing time, but up to 24 samples can be processed in parallel with the use of the associated vacuum manifold. All other kits use less plasma input and have shorter processing times, even when processing 


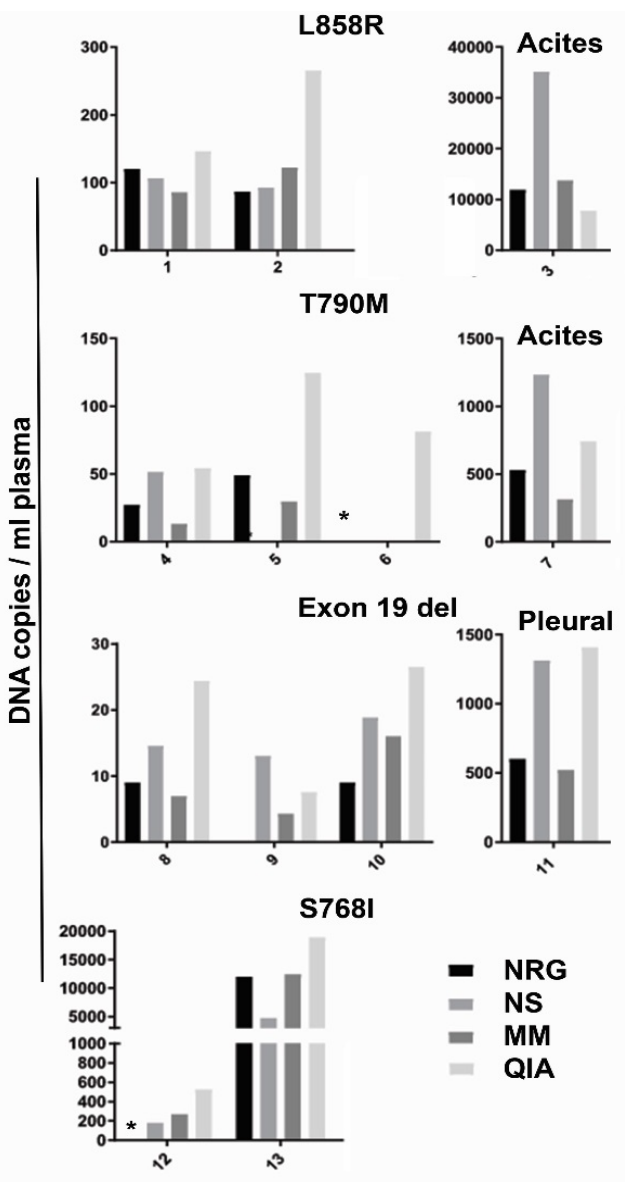

Figure 4: Mutant ctDNA detection by ddPCR. Detection of plasma ctDNA (left) and ascites or pleural fluid derived tumor DNA (right) is illustrated for the various patient samples and their applicable mutant assay. * NRG DNA extracts considered failed for sample 6 and 12, due to lack of wt ctDNA detection by ddPCR (see also Figure 3).

multiple parallel samples, as they rely on convenient spin columns or magnetic bead technology.

For all tested kits, our bio-analyser analysis data agree with previous findings that the dominant proportion of cf/ctDNA is of an approximate fragment length of around 170 base pairs, which is thought to originate from apoptotic cleavage sites defined by DNA histone occupancy [20]. The height of this peak did not reflect efficiency of DNA detection by ddPCR. Notably however, the peak heights of the assay included common markers varied for bio-analyser outputs far beyond what might be explained as pipetting errors. Although only two sets of ctDNAs isolated from two different patients could be compared by bio-analyser, these effects on the markers appeared consistent for the various kits. This might indicate presence of some components that effect bio-analyser fluorescent DNA detection in some of the kits' chemistries. Coincidently, the BIO kit appeared most affected (lowest marker peak heights), while being also incompatible with ddPCR analysis in our hands. Failure to perform well with ddPCR has been reported for other ctDNA extraction kits previously, and is important as ddPCR is becoming adopted for ctDNA testing at least in the research setting [17]. In contrast, bio-analyser data for the QIA kit produced the highest marker peak heights and, despite in comparison to the marker producing the lowest 170 base pair peak heights (i.e. according to bioanalyser the lowest cfDNA concentration), it consistently produced the highest specific DNA detection by ddPCR. Thus, interpretation of bio-analyser results remains qualitative for this study. Regardless, the data indicate that DNA extraction strategy has to be carefully tested for compatibility with the desired down-stream analysis and versatile kits that perform well with a range of applications are preferable.

Previous studies comparing cfDNA extraction methods often focussed only on DNA concentration and integrity while detection of cancer biomarkers was no or a minor focus. The most comprehensive comparison of cfDNA extraction kits performed comparison of eleven extraction methods, some not available in kit form. The study compared DNA concentration and integrity by various methods, and several extraction kits including the QIA and NRG kits, which we used in our study, performed well. However, cancer associated biomarker detection was not evaluated [11]. Also, the QIA kit as well as the DSP Virus/Pathogen Midi Kit (Qiagen) and the PME free-circulating DNA Extraction Kit (Analytik Jena) yielded various detectable DNA concentrations using a quantitative PCR approach [12].

When cfDNA was extracted in another study from serum and plasma of colorectal cancer patients, different extraction methods, the Maxwell RSC ccfDNA Plasma kit (Promega) and the QIA kits, performed well and yielded slight differences in DNA concentration and fragments and similar results for KRAS mutation detection. However mutation detection comparisons were performed on naked DNA spiked into healthy blood; naked DNA is different from ctDNA in that it lacks protein occupancy which may affect stability and extraction [13].

On a cohort of 56 patients with various cancers, two cfDNA extraction kits were compared, the MagNA Pure Compact (MPC) Nucleic Acid Isolation Kit I and the Maxwell RSC (MR) ccfDNA Plasma Kit. For only four of the plasma samples, the QIA kit used in our study was also included. While some differences in DNA concentration and integrity were detected by bio-analyser, all kits were able to detect clinically verified mutations in EGFR or KRAS for 19, or for QIA 4 plasma samples [14].

When QIA and NS kits were compared with the QIAamp DBM kit the and FA kit (EpiGentek) the QIA kit again outperformed the other kits although the DBM and NS were also able to detect exogenous DNA spiked into healthy donor plasma, whereas an earlier study of the same kits found that both Qiagen kits outperformed the others $[15,16]$

Although all these studies compared an unequal range of kits and not all were able to test multiple cancer biomarkers or a relevant number of patients, there appears to be a clear trend for the QIA kit to stand out for extraction of high quality cfDNA for downstream analysis. This was also found in a more recent and more comprehensive study that compared five cfDNA extraction kits, for DNA concentration and integrity. The study was also able to confirm known KRAS mutation status in a small cohort of plasma samples, with the QIA kit and the RSC, Maxwell RSC ccfDNA Plasma Kit (Promega) performing best for those parameters [17].

Our data comparing five cfDNA extraction kits and screening for four different EGFR mutations with sensitive ddPCR assays are adding important data to previous studies, namely that the QIA extracted ctDNA allows the best detection of EGFR biomarkers. Critically, we also show that low ctDNA concentrations may preclude biomarker detection using some of the other kits. This might have implications in the clinical setting as using a less efficient kit may miss biomarker detection in some patient samples which may preclude patients from receiving biomarker associated treatment. However, it has to be emphasised that only very low ctDNA copy numbers were undetectable 
Citation: Ding PN, Ma YF, Roberts TJ, Chua W, Bray VJ, et al. (2018) Droplet Digital PCR Based Detection of EGFR Mutations in Advanced Lung Cancer Patient Liquid Biopsies: A Comparison of Circulating Tumour DNA Extraction Kits. J Mol Biomark Diagn 9: 397. doi: 10.4172/21559929.1000397

Page 6 of 6

in several instances by a number of tested kits in our study. To date, we cannot predict what concentration of ctDNA is most informative in predicting a patient's response to therapy, disease progression or resistance to therapy. Therefore, it is still possible that relevant ctDNA concentrations may well be detectable by more economic extraction kits with easier and faster processing. Regardless, if enough plasma is available, a kit that is most sensitive in picking up cancer associated biomarkers, such as the QIA kit, would clearly be preferable. Larger prospective studies evaluating baseline and changes of ctDNA for different malignancies will provide more information regarding the clinical significance of absolute concentrations of ctDNA as measured by biomarker detection, which will aid consensus for a common ctDNA extraction protocol for clinical purposes. Interestingly, if using cfDNA extraction kits to isolate tumour DNA from other sources such as ascites and pleural fluid the MM kit appears to be the most efficient, likely due to higher total DNA concentrations.

\section{Conclusion}

The most reliable ctDNA extraction kit in our hands, for sensitive detection of EGFR mutations by ddPCR was the Qiagen circulating nuclei acid kit. Other kits missed EGFR mutations in some samples, but performed satisfactorily overall and were more economic, easier to use and effective on smaller plasma volumes. One kit was unsuitable for ddPCR down-stream analysis.

\section{Acknowledgements}

This work was supported by the Lung Foundation Australia. PD is funded by an Australian Postgraduate Scholarship through Western Sydney University and by a top up scholarship from the Cancer Institute NSW (CINSW) funded Centre for Oncology Education and Research Translation (CONCERT). TB and YM are also funded through CONCERT. TR is a CINSW FRL fellow and DL receives an Ingham Institute's Directors Scholarship. Ethics approval HREC/13/LPOOL/158 were managed through the CONCERT Biobank.

\section{References}

1. Butler TM, Spellman PT, Gray J (2017) Circulating-tumour DNA as an early detection and diagnostic tool. Curr Opin Genet Dev 42: 14-21.

2. Schwarzenbach H, Stoehlmacher J, Pantel K, Goekkurt E (2008) Detection and monitoring of cell-free DNA in blood of patients with colorectal cancer. Ann N Y Acad Sci 1137: 190-196.

3. Vendrell JA, Mau-Them FT, Beganton B, Godreuil S, Coopman P, et al. (2017) Circulating cell free tumor DNA detection as a routine tool for lung cancer patient management. Int J Mol Sci 18: 264

4. Madhavan D, Wallwiener M, Bents K, Zucknick M, Nees J, et al. (2014) Plasma DNA integrity as a biomarker for primary and metastatic breast cancer and potential marker for early diagnosis. Breast Cancer Res Treat 146: 163-174.

5. Gray ES, Rizos H, Reid AL, Boyd SC, Pereira MR, et al. (2015) Circulating tumor DNA to monitor treatment response and detect acquired resistance in patients with metastatic melanoma. Oncotarget 6: 42008-42018.

6. Gao Y, Zhang K, Xi H, Cai A, Wu X, et al. (2017) Diagnostic and prognostic value of circulating tumor DNA in gastric cancer: A meta-analysis. Oncotarge 8: $6330-6340$

7. Spindler KG, Boysen AK, Pallisgard N, Johansen JS, Tabernero J, et al. (2017) Cell-free DNA in metastatic colorectal cancer: A systematic review and metaanalysis. Oncologist 22: 1049-1055.

8. Soria JC, Ohe Y, Vansteenkiste J, Reungwetwattana T, Chewaskulyong B, et al (2018) Osimertinib in untreated EGFR-mutated advanced non-small-cell lung cancer. N Engl J Med 378: 113-125

9. Remon J, Caramella C, Jovelet C, Lacroix L, Lawson A et al (2017) Osimertinib benefit in EGFR-mutant NSCLC patients with T790M-mutation detected by circulating tumour DNA. Ann Oncol 28: 784-790.

10. Jenkins S, Yang JC, Ramalingam SS, Yu K, Patel S, et al. (2017) Plasma ctDNA analysis for detection of the EGFR T790M mutation in patients with advanced non-small cell lung cancer. J Thorac Oncol 12: 1061-1070.

11. Mauger F, Dulary C, Daviaud C, Deleuze JF, Tost J (2015) Comprehensive evaluation of methods to isolate, quantify, and characterize circulating cell-free DNA from small volumes of plasma. Anal Bioanal Chem 407: 6873-6878.

12. Sherwood JL, Corcoran C, Brown H, Sharpe AD, Musilova M, et al. (2016) Optimised pre-analytical methods improve KRAS mutation detection in circulating tumour DNA (ctDNA) from patients with Non-Small Cell Lung Cancer (NSCLC). PloS one 11: e0150197.

13. Kloten V, Ruchel N, Bruchle NO, Gasthaus J, Freudenmacher N, et al. (2017) Liquid biopsy in colon cancer: Comparison of different circulating DNA extraction systems following absolute quantification of KRAS mutations using intplex allele-specific PCR. Oncotarget 8: 86253-86263.

14. Perez-Barrios $C$, Nieto-Alcolado I, Torrente M, Jimenez-Sanchez C, Calvo V, et al. (2016) Comparison of methods for circulating cell-free DNA isolation using blood from cancer patients: Impact on biomarker testing. Transl Lung Cancer Res 5: 665-672.

15. Devonshire AS, Whale AS, Gutteridge A, Jones G, Cowen S, et al. (2014) Towards standardisation of cell-free DNA measurement in plasma: Controls for extraction efficiency, fragment size bias and quantification. Anal Bioanal Chem 406: 6499-6512.

16. Page K, Guttery DS, Zahra N, Primrose L, Elshaw SR, et al. (2013) Influence of plasma processing on recovery and analysis of circulating nucleic acids. PloS one 8: e77963.

17. Sorber L, Zwaenepoel K, Deschoolmeester V, Roeyen G, Lardon F, et al (2017) A comparison of cell-free DNA isolation kits: Isolation and quantification of cell-free DNA in plasma. J Mol Diagn 19: 162-168.

18. Ma Y, Luk A, Young FP, Lynch D, Chua W, et al. (2016) Droplet digital PCR based Androgen Receptor Variant 7 (AR-V7) detection from prostate cancer patient blood biopsies. Int J Mol Sci 17: E1264.

19. Oxnard GR, Paweletz CP, Kuang Y, Mach SL, O'Connell A, et al. (2014) Noninvasive detection of response and resistance in EGFR-mutant lung cancer using quantitative next-generation genotyping of cell-free plasma DNA. Clin Cancer Res 20: 1698-1705.

20. Jiang P, Chan CW, Chan KC, Cheng SH, Wong J, et al. (2015) Lengthening and shortening of plasma DNA in hepatocellular carcinoma patients. Proc Natl Acad Sci USA 112: E1317-1325. 